\title{
Surgery in Retroperitoneal Soft Tissue Sarcoma: A Call for a Consensus Between Europe and North America
}

\author{
Alessandro Gronchi, $\mathrm{MD}^{1}$ and Raphael Pollock, $\mathrm{MD}^{2}$ \\ ${ }^{1}$ Sarcoma Service, Department of Surgery, Istituto Nazionale Tumori, Milan, Italy; ${ }^{2}$ Division of Surgery, Department of \\ Surgical Oncology, University of Texas MD Anderson Cancer Center, Houston, TX
}

What is the proper approach to retroperitoneal sarcoma (RPS)? Can we define what is adequate and what is not? A debate on this issue between several European and North American experts has taken place in the literature during the past several years. Two possibly quite different approaches have been proposed that converge on a shared goal: to define the best strategy for patients everywhere. The article by Mussi et al. lends credence to several alternative perspectives. ${ }^{1}$ We would like to offer the reader these different views for consideration and make a suggestion regarding how to arrive at a consensus.

\section{ALESSANDRO GRONCHI: EXTENDED SURGERY IS CRITICAL TO IMPROVE OUTCOME}

Even more than that of the extremities and trunk wall, surgery of RPS often is challenging in that local recurrence affects a substantially higher proportion of patients, eventually leading to death. During the past few years, some authors have advocated the need for a more extended surgical resection strategy to improve locoregional control. $^{2,3}$ Mussi et al. add new evidence to support the need to systematically offer such an approach to all patients affected by primary RPS. ${ }^{1}$

They have found that $60 \%$ of resected adjacent organs were microscopically infiltrated by the tumor. Indeed, their surgical policy has changed during the years. It may be that organs resected in earlier time periods were only those overtly involved by the tumor, which this could have affected their high rate of infiltration. Since the shift in surgical approach more recently, favoring a wider resection

(C) Society of Surgical Oncology 2011

Published Online: 3 May 2011

A. Gronchi, MD

e-mail: alessandro.gronchi@istitutotumori.mi.it involving all organs adjacent to the tumor, the percent of affected organs would be expected to decrease. Only one other study addressing this topic has been published: Russo et al., analyzing only the kidney, found that the $20 \%$ rate of infiltration was indeed lower. ${ }^{4}$ This difference may be explained by the difference in case selection. However, Mussi et al. utilized a meticulous pathological protocol to sample the surgical specimen and study organs involvement. This could in part explain the higher rate of infiltration compared with the series by Russo et al. The high rate of microscopic infiltration may be one of the causes of the high incidence of local failure after simple excisions. A similar study performed prospectively on patients receiving a systematic extended resection might add more insight into this controversy and would be more than warranted. Nevertheless, whatever the rate of infiltration that is found, microscopic invasion of adjacent organs happens and inevitably supports a policy of extended surgery beyond visible infiltrations.

Given our knowledge of extremity lesions, which share the same pseudocapsule and pushing growth as RPS, it should come as no surprise that extensive microscopic invasion is noted in RPS. Because the microscopic invasion of adjacent organs cannot be anticipated by any means so far available, a systematic resection of clinically uninvolved adjacent viscera is the only way to clear the tumor from the affected site. Extremity lesions are routinely treated with wide resection, and the impact of inadequate margins on outcome has been extensively studied. ${ }^{5-8}$ Every attempt is made to avoid positive surgical margins.

Why should the same concept not be applied in RPS? Tissues surrounding RPS are visceral organs rather than muscles. Therefore, an attempt to resect RPS surrounded by healthy tissue inevitably requires the resection of adjacent viscera, involved or not $(74 \%$ of the patients had $\geq 2$ organs resected in our series ${ }^{9}$ ). Once we accept this, we 
should carefully balance the morbidity related to multivisceral resection and the expected benefit. In fact, although wide surgery is universally accepted for extremity sarcomas, care is taken with respect to critical structures, such as vessels and nerves; we dissect vessels under adventitia and nerve under epineurium and we resect them only if directly infiltrated. This also could apply to RPS surgery, including vessels/nerves as well as some visceral organs. Although the same quality of margins as in limb sarcomas may be difficult to reach in the retroperitoneum, nonetheless it can be approximated. In addition, due to the variety of presentations, in some patients the quality of margins will be better and worse in others; however, this should not prevent the latter cohort from receiving an attempt at wide resection. Given the complexity and rarity of the disease, we strongly recommend referral of these patients to specialized centers. Surgeons in the community should not attempt to resect these tumors in a limited or extended manner.

Another finding in favor of a liberal approach to primary disease is the high rate of residual disease found by Bonvalot in a series of 21 patients who underwent reexcision in the absence of macroscopic disease $(80 \%){ }^{2}$ Although we cannot recommend systematic reexcision of all patients treated by simple resection, her finding supports the need of an initial extended approach to better clear the tumor from the retroperitoneal space and possibly increase the rate of cure.

There is retrospective evidence that the extended approach translates into far better local control (approximating $80 \%$ at 5 years) as recently reported. ${ }^{2,3}$ This improvement in local outcome has been showed for low-, intermediate-, and high-grade lesions. Because histological grade of aggressiveness is the major outcome prognosticator in all series, it is highly probable that the extended approach will be critical for curing low- and intermediategrade tumors, whereas it will need to be complemented by other therapies in high-grade lesions. The price to pay is the extended procedure described, involving adjacent uninvolved organs, which indeed may have some added morbidity. Yet recent reports have shown the safety and relatively low morbidity if these procedures are performed in high-volume centers. ${ }^{9,10}$ These findings should be interpreted as reinforcing the message that these tumors should be referred to specialized centers to maximize the efficacy ratio between the necessity to achieve wide margins and the expected morbidity. Time will tell whether the benefit in local control achieved with extended surgical approach will translate into a long-term survival benefit.

Preliminary evidence in support of this position was recently reported at the 2010 meeting of the Connective Tissue Oncology Society in Paris. This is not surprising in that it is well known for extremity sarcoma that the quality of initial surgery in addition to biology accounts for the difference in sarcoma long-term survival for each histological subtype and grade. ${ }^{11}$

We acknowledge that we are speculating on retrospective data and we have to be cautious given the well-known biases inevitable in such analyses. However, ultimately all knowledge about the effects of surgery is retrospective. Moreover, attempting to study these tumors by means of prospective, randomized trials would be problematic, especially if stratifying by subgroup, given that the incidence of retroperitoneal sarcoma is $<0.5 / 100,000$ per year.

\section{RAPHAEL POLLOCK: RETROPERITONEAL SARCOMA HISTOLOGICAL SUBTYPE HELPS TO INFORM SURGICAL RESECTION STRATEGY}

Several critical issues are identified in the intriguing report from Mussi et al., in which a retrospective, singleinstitutional series of primary retroperitoneal soft tissue sarcomas $(1996-2008 ; \mathrm{n}=77)$ is presented as providing evidence that a universal policy of wide margin multivisceral resection is warranted for this surgically challenging disease entity. Indeed, the very title of the manuscript, "Retroperitoneal Sarcoma: Is it Time to Change the Surgical Policy?" implies that the current modus operandi is no longer applicable or perhaps even acceptable. Before delving into the arguments and supporting evidence, it is probably pertinent to question whether any knowledgeable student of this disease currently advocates a global policy of wide field multivisceral resection for all retroperitoneal sarcoma patients any more than a practice encompassing minimalist resection strategies for all such afflicted individuals. Unequivocally, the answer to either of these surgical extremes must be a rejection of both approaches in favor of the prospective, personalized application of all relevant modalities, including systemic treatments, radiotherapy, and (extent of) surgical interventions.

Mussi raises several issues that merit comment. It is interesting that the most common tumor-adjacent "organ" resected was the psoas muscle. It is not clear whether such resections included the entire psoas muscle (and therefore mandatory proximal femoral nerve sectioning) or whether a partial resection was performed. If the latter, it would be interesting to learn more about the basis upon which the borders of resection were determined intraoperatively: if less than an entire organ was resected then the possibility of tumor transsection with potential microscopic tumor bed contamination would certainly be a concern. It also would be interesting to learn more about just how wide a margin would be needed to prospectively satisfy oneself that an adequate margin of resection had been achieved. Although this is a difficult parameter to unambiguously quantify, 
I suggest that this is nonetheless the responsibility of those advocating a universal wide margin multivisceral resection management policy.

What were the consequences of the resection policy advocated by Mussi? Approximately $40 \%$ of the adjacent resected organs did not have detectable macro- or microscopic sarcoma on final pathologic analysis, whereas $25 \%$ of final pathology reports demonstrated tumor perimeter microscopic positivity, pointing to the inherent inability of preoperative radiologic studies or intraoperative adjacent organ visual inspection to establish prospectively final R0 or $\mathrm{R} 1$ resection margin status. So, how to intraoperatively decide on the extent of adjacent organ resection? No doubt there would be agreement that a sleeve resection of a tumor-adherent segment of terminal ileum is an entirely different circumstance than resection of a tumor-adherent duodenal sweep/head of the pancreas confluence. Some specific guidance is needed: intraoperatively, how wide will be wide enough?

Can we resolve these dilemmas? We now know that all retroperitoneal sarcomas are not created equal. Unequivocally, the biology of the major histopathological subtypes is remarkably different, spanning the spectrum from avid and rapid metastasis (leiomyosarcoma) to indolent local recurrence essentially devoid of metastatic capacity (welldifferentiated liposarcoma). ${ }^{12}$ In fact, these biological differences are components of several retroperitoneal sarcoma predictive nomograms, which leverage histologically defined survival rate variability in the determination of overall prognosis. ${ }^{13,14}$ It is interesting that the vast majority of long-term retroperitoneal sarcoma survivors (i.e., those with greater than 10-year disease-specific survival) are those with (well-differentiated) liposarcoma, an observation first made at least 20 years ago. ${ }^{15}$

This reality must inform our decisions about extent of resection, especially for well-differentiated liposarcoma. After all, is aggressive resection of contiguous organs, $40 \%$ of which will prove to be microscopically sarcoma-free, always the best initial surgical option for a patient whose tumor biology typically portends a long term, metastasisfree survival as the bearer of a chronic, multiply locally recurrent, multifocal disease process? The diffuse pattern of failure for well-differentiated liposarcoma (i.e., failure throughout the retroperitoneum, including areas remote from the original surgical site) would suggest that any fatbearing tissue in the retroperitoneum might emerge as the locus of subsequent recurrence. Does this mandate a policy of complete resection of all retroperitoneal fatty tissues when operating a patient with well differentiated liposarcoma? Even if we agreed that such an approach would confer optimal disease control for this subset of retroperitoneal sarcoma patients, is it truly achievable?
As Murray Brennan has pointed out repeatedly, after controlling for surgical intervention performed at a highest possible level of quality, tumor biology ultimately drives both local and distant sarcoma recurrence. ${ }^{16}$ The validity of this conception is borne out by examination of 5-year overall survival percentages in retroperitoneal sarcoma series: slightly less than $75 \%$ in the Mussi series, approximately $80 \%$ in the Bonvalot and the Gronchi series, ${ }^{2,3}$ and approximately $64 \%$ for non-well-differentiated liposarcoma patients versus $95 \%$ for the well-differentiated liposarcoma subset in the UTMDACC series. ${ }^{17}$ Are these differences significant? Perhaps; however, I would anticipate that disease-free survival at 10 years would probably be more or less equivalent across centers of excellence in that the toll of surgical morbidity and unfavorable histological subtype would have already been paid long before, leaving a residuum of long-term survivors who will predominantly have more indolent retroperitoneal sarcoma tumor biology, most commonly well-differentiated liposarcoma.

A cautionary note is in order: in considering the abovementioned results from several European and American centers of excellence, it is unlikely that the above-mentioned survival differences are due to the inherent quality or extent of surgery performed at one center versus another. Allowing histology to inform decisions about extent of resection is always trumped by the need and desire to achieve an $\mathrm{R} 0$ or at least an $\mathrm{R} 1$ resection regardless of histological subtype, even if that necessitates contiguous organ resection - a very common occurrence on this side of the Atlantic Ocean as well (69\% of all retroperitoneal sarcoma resections in the UTMDACC series). ${ }^{17}$ Instead, such differences-if indeed they are real-most likely reflect differences in underlying histology subtype-defined sarcoma biology rather than quality or extent of surgery per se. Unequivocally, we need better tools to define the normal tissue:tumor interface during surgery; hopefully in the future the intraoperative application of "cutting edge" new diagnostics may help us decide just how "wide" we need to go! $!^{18}$

\section{HOW TO ACHIEVE CONSENSUS (ALESSANDRO GRONCHI AND RAPHAEL POLLOCK)}

It is an unfortunate reality that prospective, randomized retroperitoneal sarcoma clinical trials to test these contentions are probably an unrealistic (unrealizable?) aspiration, at least given historical experiences. Novel intraoperative diagnostic tools remain a future, albeit distant, likelihood. What we can do now is establish comprehensive prospective registries with standardized guideline definitions of the extent of surgical resection, then have the courage to 
openly compare our results as tabulated over time. By means of an example, such an "open" approach is currently underway in a shared attempt to establish the most robust retroperitoneal sarcoma nomogram in which the MSKCC, UTMDACC, and NCI/Milano retroperitoneal sarcoma algorithms are being compared for prognostic efficacy using a large-scale amalgamated dataset incorporating information from these and other major sarcoma centers. Another example are the sarcoma wet clinics annually being conducted under the leadership of Sylvie Bonvalot at Institute Gustave Roussy in which sarcoma surgical oncologists from many countries are having the opportunity to work together in the operating room. The world is shrinking, and we should aggressively embrace (and create!) these and other opportunities to learn from each other, ultimately to the benefit of patients everywhere.

\section{REFERENCES}

1. Mussi C, Colombo P, Bertuzzi A, et al. Retroperitoneal sarcoma: is it time to change surgical policy? Ann Surg Oncol. (in press).

2. Bonvalot S, Rivoire M, Castaing M, et al. Primary retroperitoneal sarcomas: a multivariate analysis of surgical factors associated with local control. J Clin Oncol. 2009;27:31-7.

3. Gronchi A, Lo Vullo S, Fiore M, et al. Aggressive surgical policies in a retrospectively reviewed single-institution case series of retroperitoneal soft tissue sarcoma patients. J Clin Oncol. 2009; 27:24-30.

4. Russo P, Kim Y, Ravindran S, et al. Nephrectomy during operative management of retroperitoneal sarcoma. Ann Surg Oncol. 1997;4(5):421-4.

5. Stojadinovic A, Leung DH, Hoos A, et al. Analysis of the prognostic significance of microscopic margins in 2,084 localized primary adult soft tissue sarcomas. Ann Surg. 2002;235:424-34.
6. Gronchi A, Lo Vullo S, Colombo C, et al. Extremity soft tissue sarcoma in a series of patients treated at a single institution: local control directly impacts survival. Ann Surg. 2010;251:512-7.

7. Trovik CS, Bauer HC, Alvegard TA, et al. Surgical margins, local recurrence and metastasis in soft tissue sarcomas: 559 surgicallytreated patients from the Scandinavian Sarcoma Group register. Eur J Cancer. 2000;36(6):710-6.

8. Zagars GK, Ballo MT, Pisters PWT, et al. Prognostic factors for patients with localized soft-tissue sarcoma treated with conservation surgery and radiation therapy. Cancer. 2003;97:2530-43.

9. Bonvalot S, Miceli R, Berselli M. Aggressive surgery in retroperitoneal soft tissue sarcoma carried out at high-volume centers is safe and is associated with improved local control. Ann Surg Oncol. 2010;17:1507-14.

10. Tseng WH, Martinez SR, Tamurian RM, et al. Contiguous organ resection is safe in patients with retroperitoneal sarcoma: an ACS-NSQIP analysis. J Surg Oncol. 2011;103(5):390-4.

11. Stojadinovic A, Leung DHY, Allen P, et al. Primary adult soft tissue sarcoma: time-dependent influence of prognostic variables. J Clin Oncol. 2002;20:4344-52.

12. Lewis JJ, Leung D, Woodruff JM, et al. Retroperitoneal softtissue sarcoma: analysis of 500 patients treated at a single institution. Ann Surg. 1998;228:355-65.

13. Ardoino I, Miceli R, Berselli M, et al. Histology-specific nomogram for primary retroperitoneal soft tissue sarcoma. Cancer. 2010;116:2429-36.

14. Anaya DA, Lahat G, Wang X, et al. Postoperative nomogram for survival of patients with retroperitoneal sarcoma treated with curative intent. Ann Oncol. 2009;249:1014-22.

15. Storm FK, Mahvi DM. Diagnosis and management of retroperitoneal soft-tissue sarcoma. Ann Surg. 1991;214:2-10.

16. Brennan MF. The enigma of local recurrence. Ann Surg Oncol. 1997;4:1-12.

17. Anaya DA, Lahat G, Wang X, et al. Establishing prognosis in retroperitoneal sarcoma: a new histology-based paradigm. Ann Surg Oncol. 2009;16:667-75.

18. Carbajal EF, Baranov SA, Young ED, et al. Revealing retroperitoneal liposarcoma morphology using optical coherence tomography. J Biomed Optics. 2011;16:205021-3. 